Original paper

\title{
Prevalence and clinical profile of glycogen storage diseases in children from Western India
}

\author{
Ira Shah, Drishti Tolani, Naman S Shetty, Vishrutha Karkare \\ Pediatric Liver Clinic, Department of Pediatric Gastroenterology and Hepatology, B J Wadia Hospital for Children, Mumbai, India
}

\begin{abstract}
Aim of the study: To determine the prevalence and clinical profile of glycogen storage diseases (GSD) in children in western India.

Material and methods: This retrospective analysis was conducted over a period of 7 years from 2006 to 2012. All children diagnosed with GSD on liver biopsy were included in the study. Their clinical history, examination, biochemical profile and outcome were noted.

Results: Of 751 children, 18 (2.4\%) were clinically diagnosed with GSD. Mean age at presentation was $2.3 \pm 1.3$ years. Male : female ratio was $1: 1$. The main presenting features were abdominal distension in $15(83.3 \%)$ patients, hepatomegaly in all $18(100 \%)$, splenomegaly in $11(61.1 \%)$ and jaundice in $2(11.1 \%)$ patients. Four (22.2\%) patients had delayed development. Four (22.2\%) patients were siblings to an older affected child. Only $1(5.6 \%)$ patient had portal hypertension and $2(11.1 \%)$ had ascites. Only $1(5.6 \%)$ patient had elevated bilirubin levels, 17 (94.4\%) had elevated serum glutamic-oxaloacetic transaminase (SGOT) and all 18 (100\%) patients had elevated serum glutamic-pyruvic transaminase (SGPT) levels. Nine (69.3\%) patients of the 13 tested had acidosis, $1(9.1 \%)$ had elevated uric acid, $2(11.1 \%)$ had neutropenia, 8 (44.4\%) experienced hypoglycemia, $4(22.2 \%)$ patients had nephromegaly and only 1 patient showed evidence of cirrhosis in the liver biopsy. Fifteen (83.3\%) patients were short. Three out of 6 patients tested had hypertriglyceridemia (50\%). One (5.6\%) patient died, $9(50 \%)$ patients were lost to followup and the remaining 8 (44.4\%) patients continued regular follow-up.

Conclusions: Metabolic acidosis, hypertriglyceridemia, short stature, and hypoglycemia are major problems in children with GSD. Most of the patients are referred late at the time of presentation.
\end{abstract}

Key words: children, India, glycogen storage disease.

\section{Address for correspondence}

Prof. Dr. Ira Shah, Head, Department of Pediatric Gastroenterology and Hepatology, B J Wadia Hospital for Children, Parel, Mumbai 400012, India, e-mail: irashah@pediatriconcall.com

\section{Introduction}

Glycogen storage diseases (GSD) are inherited metabolic disorders involving defects in the enzymes responsible for glycogen synthesis and breakdown [1]. There are 11 distinct types of GSD based on different enzyme deficiencies [1]. GSD primarily affect the liver, muscle, heart and sometimes the central nervous system [1]. GSD are usually diagnosed in infancy or early childhood with hypoglycemia, hepatomegaly, poor physical growth and deranged biochemical profile [2]. The overall frequency of GSD in the United States has been estimated to be 1 per 20-25,000 births [3] and according to British Columbia it has been estimated to be 1 in every 40,000 [4]. No large scale study estimating the incidence of GSD in India has been recorded in the literature, but a study conducted in South India recorded clinical phenotypes of 17 cases [5]. Individual case reports of the various types of GSD have also been recorded in the literature. We undertook this study to determine prevalence of GSD among children with liver diseases in western India and determine the clinical profile of these patients.

\section{Material and methods}

A retrospective analysis of children with GSD was done after hospital academic council approval. Re- 
cords of all patients who were referred to a pediatric liver clinic within the period May 2006 to December 2012 were reviewed. All patients diagnosed with GSD on the basis of liver biopsy (histopathology showing glycogen laden cells; PAS (periodic acid-Schiff) positive and diastase sensitive) were included in the study. Subtype of GSD could not be determined as enzyme estimation on liver biopsy could not be done due to non-availability at the time of liver biopsy. All patients had undergone complete blood count, blood sugar estimation, liver function tests and blood gas levels. Records of serum lactic acid, uric acid and triglycerides were present only in some cases. Patients were suspected to have GSD type I if they had hyperlactemia, hyperlipidemia, hyperuricemia with/without hypoglycemia. Patients were suspected to have GSD type Ib if they additionally had neutropenia. Patients were suspected to have GSD type III if they had hepatomegaly with/without hypoglycemia along with hyperlipidemia without hyperuricemia or hyperlactatemia. Pompe's disease was diagnosed if there was associated myopathy and cardiomyopathy. Growth records were noted and patients were determined to be malnourished if weight was less than the $5^{\text {th }}$ centile and stunted if height was less than the $5^{\text {th }}$ centile as per Agarwal's charts [6]. Development was determined by Denver's scale [7]. Portal hypertension was determined if portal vein size was more than $1 \mathrm{~cm}$ on color Doppler of the portal venous system or there were collaterals on color Doppler or esophagogastroscopy (OGD scopy) showed varices. Bilirubin was considered to be elevated if serum bilirubin was more than $1.2 \mathrm{mg} / \mathrm{dl}$. Serum glutamic-oxaloacetic transaminase (SGOT) was considered to be elevated if it was more than $40 \mathrm{IU} / \mathrm{l}$ and serum glutamic-pyruvic transaminase (SGPT) was

Table 1. Biochemical profile of all patients at time of presentation

\begin{tabular}{lc}
\hline Parameter & Mean \pm SD \\
\hline Bilirubin (mg/dl) & $1.2 \pm 1.7$ \\
\hline SGOT (IU/l) & $294.5 \pm 255.9$ \\
\hline SGPT (IU/l) & $247.8 \pm 172.5$ \\
\hline Albumin (gm/dl) & $3.9 \pm 1.4$ \\
\hline PT (s) & $12.6 \pm 1.7$ \\
\hline PTT (s) & $29.1 \pm 8.7$ \\
\hline Lactate $(\mathrm{mg} / \mathrm{dl})$ & $28.8 \pm 33.3$ \\
\hline Bicarbonate $(\mathrm{mmol} / \mathrm{l})$ & $14.7 \pm 1.8$ \\
\hline Uric acid $(\mathrm{mg} / \mathrm{dl})$ & $4.2 \pm 2.1$ \\
\hline Neutrophil count $\left(\mathrm{cell} / \mathrm{s} / \mathrm{mm}^{3}\right)$ & $9217.2 \pm 15974.03$ \\
\hline Blood sugar $(\mathrm{mg} / \mathrm{dl})$ & $91 \pm 34.7$ \\
\hline
\end{tabular}

SGOT - serum glutamic-oxaloacetic transaminase, SGPT - serum glutamic-pyruvic transaminase, PT - prothrombin time, PTT - partial thromboplastin time considered elevated if it was more than $25 \mathrm{IU} / \mathrm{l}$. Hypoalbuminemia was determined if serum albumin was less than $3.5 \mathrm{gm} / \mathrm{dl}$. Prothrombin time (PT) more than 2 seconds above control and partial thromboplastin time (PTT) more than 9 seconds above control were considered to be prolonged. Metabolic acidosis was determined if $\mathrm{pH}$ was less than 7.3 and bicarbonate was less than $22 \mathrm{mmol} / \mathrm{l}$. Hypoglycemia was determined if blood sugar was less than $60 \mathrm{mg} / \mathrm{dl}$. Neutropenia was determined if absolute neutrophil count was $<1500$ cells $/ \mathrm{mm}^{3}$. Serum uric acid was elevated if more than $7 \mathrm{mg} / \mathrm{dl}$ and triglycerides more than $200 \mathrm{mg} / \mathrm{dl}$ were considered to be elevated. Nephromegaly was determined if kidney sizes on ultrasound were more than the reference values for age. Outcome of these patients was recorded.

\section{Results}

From a total of 751 cases referred to the clinic, $18(2.4 \%)$ children were clinically diagnosed with GSD with 5 patients suspected to have type I of which 2 were type Ib with neutropenia. Three patients were suspected to have type III GSD and the remainder remained unclassified. No patient was suspected to have GSD type II. Liver biopsy showed plant cell morphology with ballooned hepatocytes containing glycogen, PAS positive in 15 patients and early fibrosis in 3 patients. There was cirrhosis in one patient. Mean age at presentation was $2.3 \pm 1.3$ years, with range of 0.3 months to 5 years. Male : female ratio was $1: 1$. The main presenting features were abdominal distension in $15(83.3 \%)$ patients, large hepatomegaly in all $18(100 \%)$, splenomegaly in $11(61.1 \%)$ patients and jaundice in $2(11.1 \%)$ patients. Four (22.2\%) patients had delayed development. Four (22.2\%) patients were siblings to an older affected child and were diagnosed on family screening. Only one (5.6\%) patient had findings of portal hypertension on color Doppler of portal venous system and $2(11.1 \%)$ patients had ascites. The biochemical profile at the time of presentation is depicted in Table 1. Only 1 (5.6\%) patient had elevated bilirubin levels, 17 (94.4\%) had elevated SGOT and all $18(100 \%)$ patients had elevated SGPT levels. Of the 13 patients in whom serum albumin records were available, $3(23.1 \%)$ patients were found to have hypoalbuminemia. PT and PTT levels were recorded in 14 patients, out of which $4(28.5 \%)$ patients had elevated PT and $2(14.3 \%)$ patients had elevated PTT. Nine (69.3\%) patients of the 13 tested were found to have acidosis. Serum lactate levels were recorded in 5 patients out of which $2(40 \%)$ patients had elevated levels. Of the 11 patients in whom the serum uric acid levels 
were recorded, only 1 (9.1\%) had elevated uric acid. Only $2(11.1 \%)$ patients were found to have neutropenia, $8(44.4 \%)$ patients experienced hypoglycemia and $4(22.2 \%)$ patients had findings of nephromegaly. Fifteen $(83.3 \%)$ patients were found to be short. None of the patients were found to have cardiomyopathy and only $3(16.7 \%)$ patients were found to suffer from seizures. Three out of 6 patients had hypertriglyceridemia (50\%). On follow-up, 1 (5.6\%) patient died at the age of 2 years due to hepatic encephalopathy, 9 (50\%) patients were lost to follow-up and the remaining 8 (44.4\%) patients continued regular follow-ups.

\section{Discussion}

To date in the literature no large-scale studies estimating the overall incidence of GSD in India have been recorded. A study conducted in South India reviewed 17 cases of GSD in which the mean age at presentation was 15 months [5], which is much lower than the 2.3 years recorded as the mean age in our study. All the patients presented with hepatomegaly and a distinct pattern of swollen hepatocytes. The prevalence rate of clinically detected GSD was $2.3 \%$ in our study, as determined from all children with liver diseases referred to our clinic. Thus, the actual prevalence of GSD among the general population could not be determined.

The liver in commonly involved in GSD types I, III, IV, VI and IX. According to the literature, $80 \%$ of hepatic GSD cases involve types I, III and IX [8]. Symptomatic liver involvement is most commonly seen in GSD types I and III, both of which are characterized by hypoglycemia and hepatomegaly [8]. Hypoglycemia is considered as the primary indicator of liver involvement [1]. Thus, it is possible to diagnose the type of GSD from its clinical, biochemical and histological features. However, the best diagnosis of the type of GSD can be made by enzyme studies of liver tissue obtained by biopsy [9]. In our study, all patients had hepatomegaly and hypoglycemia suggestive of either type I or III, but since we could not do enzyme estimation, we could not confirm the exact subtype of GSD. However, 2 patients did have neutropenia suggestive of subtype Ib in these patients. GSD type Ib is characterized by neutropenia and neutrophil dysfunction along with disturbed glucose homeostasis caused due to deficiency of glucose-6-phosphate transporter [10]. Such patients are susceptible to recurrent bacterial infections usually before 1 year of age [1]. None of our patients had recurrent infections.

Growth retardation is a remarkable feature in GSD for both types I and III [5] which is compatible with over $80 \%$ patients found to be of short stature in our study. Besides hypoglycemia and hepatomegaly other striking abnormalities of type I GSD include lactic acidosis, hypertriglyceridemia and hyperuricemia [5]. Similar findings were seen in our study.

During infancy, SGOT, SGPT and lactate dehydrogenase (LDH) levels are markedly elevated in type III GSD but the levels decrease with increasing age and decreasing size of the liver [5]. In our study $94.4 \%$ of patients had elevated SGOT levels and $100 \%$ of patients had elevated SGPT levels. Clinically type III GSD may present with muscle weakness, gross motor delay, hypotonia and cardiomyopathy [11]. In our study, however, none of the patients showed signs of cardiomyopathy; motor delay was seen in 1 patient and 1 patient suffered from proximal myopathy.

The treatment of GSD is type specific. Treatment for GSD type I and III is primarily dietary and aims at maintaining normal blood glucose levels with frequent meals rich in carbohydrates and corn starch $[12,13]$ so as to improve the clinical and biochemical status and increase overall life expectancy [13]. Similar diet was also prescribed in our patients as most patients were suspected to have either type I or III disease. Death is mostly due to metabolic derangement [13]. Also, in older patients progressive renal diseases and liver adenomas that arise as complications with ageing have been reported as the two major causes of morbidity and mortality in older patients [13].

\section{Conclusions}

Metabolic acidosis, hypertriglyceridemia, short stature, and hypoglycemia are major problems in children with GSD. Most of the patients are referred late at the time of presentation.

\section{Disclosure}

The authors report no conflict of interest.

\section{References}

1. Hicks J, Wartchow E, Mierau G. Glycogen storage diseases: a brief review and update on clinical features, genetic abnormalities, pathologic features, and treatment. Ultrastruct Pathol 2011; 35: 183-196.

2. Goldberg T, Slonim AE. Nutrition therapy for hepatic glycogen storage disease. J Am Diet Assoc 1993; 12: 1423-1430.

3. Chen YT. Glycogen storage diseases and other inherited disorders of carbohydrate metabolism. In: Kasper DL, Braunwald E, Fauci AS, et al. (eds.). Harrison's Principles of Internal Medicine. Mc Graw-Hill, New York 2005; 2319-2323.

4. Applegarth DA, Toone JR, Lowry RB. Incidence of inborn errors of metabolism in British Columbia, 1969-1996. Pediatrics 2000; 105: e10. 
5. Koshy A, Ramaswamy K, Correa M, Rekha S. Glycogen storage disease: report of 17 cases from southern India. Indian J Gastroenterol 2006; 25: 182-184.

6. Khadilkar VV, Khadilkar AV, Choudhury P, et al. IAP growth monitoring guidelines for children from birth to 18 years. Indian Pediatrics 2007; 44: 187-197.

7. Frankenburg WK, Dobbs JB. The Denver Developmental Screening Test. J Pediatr 1967; 71: 181-191.

8. Saltik IN, Ozen H, Ciliv G, et al. Glycogen Storage disease type Ia: frequency and clinical course in Turkish children. Indian J Pediatric 2000; 67: 497-501.

9. Jevon GP, Finegold MJ. Reliability of histological criteria in glycogen storage disease of the liver. Pediatr Pathol 1994; 14: 709-721.

10. Chou JY, Jun HS, Mansfield BC. Neutropenia in type Ib glycogen storage disease. Curr Opin Hematol 2010; 17: 36-42.

11. Sujatha J, Amithkumar IV, Lathaa B. Prenatal Diagnosis of Glycogen Storage Disorder Type III. Indian Pediatr 2010; 47: 354355.

12. Hasan Ö. Glycogen storage diseases: New perspectives. World J Gastroenterol 2007; 13: 2541-2553.

13. Rake JP, Visser G, Labrune P, et al. Glycogen storage disease type I: diagnosis, management, clinical course and outcome. Results of the European Study on Glycogen Storage Disease Type I (ESGSD I). Eur J Pediatr 2002; 161: S20-S34. 